\title{
The impact of life form on the architecture of orchid mycorrhizal networks in tropical forest
}

\section{Abstract}

Understanding the processes that determine the architecture of interaction networks represents a major challenge in ecology and evolutionary biology. One of the most important interactions involving plants is the interaction between plants and mycorrhizal fungi. While there is a mounting body of research that has studied the architecture of plant-fungus interaction networks, less is still known about the potential factors that drive network architecture. In this study, we investigated the architecture of the network of interactions between mycorrhizal fungi and 44 orchid species that represented different life forms and co-occurred in tropical forest and assessed the relative importance of ecological, evolutionary and co-evolutionary mechanisms determining network architecture. We found 87 different fungal operational taxonomic units (OTUs), most of which were members of the Tulasnellaceae. Most orchid species associated with multiple fungi simultaneously, indicating that extreme host selectivity was rare. However, an increasing specificity towards Tulasnellaceae fungal associates from terrestrial to epiphytic and lithophytic orchids was observed. The network of interactions showed an association pattern that was significantly modular $\left(M=0.7389, M_{\text {random }}=0.6998\right)$ and nested $(\mathrm{NODF}=5.53, P<0.05)$. Terrestrial orchids had almost no links to modules containing epiphytic or lithophytic orchids, while modules containing epiphytic orchids also contained lithophytic orchids. Within each life form several modules were observed, suggesting that the processes that organize orchid-fungus interactions are independent of life form. The overall phylogenetic signal for both partners in the 
24 with a wide number of mycorrhizal fungi and that ecological rather than phylogenetic constraints

25 determine network architecture.

\section{Keywords}

27 orchid life form, interaction network, modularity, nestedness, orchid mycorrhiza 
Understanding the ecological, evolutionary and co-evolutionary processes that shape the architecture of interaction networks represents one of the main challenges in ecology and evolutionary biology (Bascompte 2010). In general, two types of interaction networks have been described (summarized in Bascompte and Jordano 2014). On the one hand, interaction networks may consist of several subnetworks or modules of species that interact more with each other than with other species in the network (e.g. Olesen et al. 2007, Rezende et al. 2009, Fortuna et al. 2010, Donatti et al. 2011, Jacquemyn et al. 2015). This type of network architecture is typically encountered in species displaying antagonistic interactions, but can also be found in mutualistic et al. 2007, Thébault and Fontaine 2010).

One of the most ubiquitous interactions involving plants is the association between plants and mycorrhizal fungi. In this interaction, fungi facilitate plants with the acquisition of essential nutrients from the soil, and in return, plants generally transfer photosynthetically fixed carbon to their fungal partners (Bonfante \& Genre, 2010; van der Heijden et al., 2015). Based on a mounting body of research describing the architecture of plant-fungus interaction networks (e.g. Chagnon et al. 2012, Montesinos-Navarro et al. 2012, Bahram et al. 2014, Toju et al. 2014, 2016), it has recently been suggested that the degree of nestedness and modularity are organized along a continuous gradient that is mainly driven by nutrient properties and the level of mutualism (van der Heijden et al. 2015). In general, arbuscular mycorrhizal interaction networks tend to be nested (e.g. Chagnon et al. 2012, Montesinos-Navarro et al. 2012), whereas orchid mycorrhizal networks 
are often modular (e.g. Martos et al. 2012, Jacquemyn et al. 2015). Ectomycorrhizal networks are

51 predicted to be somewhere in between (van der Heijden et al. 2015). This simple prediction has

52 recently been challenged by Põlme et al. (2018), who summarized information on network

53 structure from a large number of studies investigating arbuscular mycorrhizal, ectomycorrhizal,

54 ericoid mycorrhizal and orchid mycorrhizal interactions. Their meta-analysis showed that the

55 degree of nestedness was not significantly affected by fungal guild, but that modularity was higher

56 in ericoid and orchid mycorrhizal fungi than in the other fungal guilds. However, the precise mechanisms responsible for generating such patterns in plant-fungus interaction networks remain remain poorly understood (Chagnon 2016; Põlme et al. 2018).

With an estimated number of $>27.000$ species, the orchid family encompasses a considerable diversity in life forms, with approximately $30 \%$ of species being terrestrial and the remaining $70 \%$ being known for their potential to explore highly stressful epiphytic and lithophytic habitats (Gravendeel et al. 2004, Dearnaley et al. 2012). Regardless of their life form, orchids invariably rely on mycorrhizal fungi for seed germination and subsequent establishment of seedlings, and most orchids retain mycorrhizal associations at adulthood as well (Rasmussen and Rasmussen 2009). When multiple orchids co-occur, they often tend to associate with different sets of mycorrhizal fungi, leading to mycorrhizal networks that are significantly modular (Martos et al. 2012, Jacquemyn et al. 2015). However, it remains unclear what factors exactly drive modularity in orchid mycorrhizal networks. patterns, their distribution is assumed to be independent of their partner plants (Smith and Read 
the availability of above-ground water and nutrient supplies decreases from terrestrial to lithophytic habitats, it can be predicted that more stressful environments may limit the occurrence and abundance of orchid mycorrhizal fungi or select for a limited set of strains that are capable of surviving in these environments. Extreme host selectivity and specialization (Taylor and Bruns 1997, Shefferson et al. 2005) may therefore to some extent explain the significant modularity typically found in orchid-fungus networks. However, many orchid species have been shown to associate with multiple fungi at the same time (Waterman et al. 2011, Jacquemyn et al. 2014, 2015), so that extreme host selectivity cannot be the sole factor explaining modularity in orchid mycorrhizal networks. The alternative hypothesis would be that modularity is driven by ecological constraints (Martos et al. 2012). In this case, variation in local growth conditions and the patchy distribution of compatible fungi may explain modularity (Jacquemyn et al. 2012, 2014). In case host selectivity and specialization are also phylogenetically conserved (Shefferson et al. 2005, Xing et al. 2017), the network of interactions and modularity should also show a significant phylogenetic signal (Jacquemyn et al. 2011). constraints on the structure of the network of interactions between orchids displaying various life forms and their mycorrhizal fungi. More specifically, we set out to investigate the following questions:

1. Does mycorrhizal fungal community composition significantly differ between orchids with different life forms? significant nestedness and/or modularity? 
3. Can the structure of observed orchid mycorrhizal network be explained by extreme host selectivity, phylogenetic or ecological constraints?

To answer these questions, we investigated mycorrhizal associations in 44 different orchid species that occurred in moist tropical forest of Xishuangbanna in the Yunnan province, China, and that displayed different life forms.

99

\section{Materials and methods}

\section{Study sites and sampling}

This study was conducted in the Xishuangbanna region $\left(21^{\circ} 8^{\prime}-22^{\circ} 36^{\prime} \mathrm{N}, 99^{\circ} 56^{\prime}-101^{\circ} 51^{\prime} \mathrm{E}\right)$ in the southern part of the Yunnan Province, China (Fig. S1). This region is biogeographically situated in a transitional zone from tropical South-east Asia to temperate East-Asia. Xishuangbanna has the largest area of tropical forest remaining in the country and contains approximately 5000 species of higher plants (Zhang and Cao 1995). This region is characterized by a semi-humid, tropical monsoon climate with annual temperatures varying between $15.1^{\circ} \mathrm{C}$ and $21.7^{\circ} \mathrm{C}$ and the annual rainfall between 1196 and $2492 \mathrm{~mm}$. A national nature reserve $\left(100^{\circ}\right.$ $16^{\prime}-101^{\circ} 50^{\prime} \mathrm{E}, 21^{\circ} 10^{\prime}-22^{\circ} 24^{\prime} \mathrm{N}$ ) was established in Xishuangbanna in 1958 , which consists of five subreserves: Mengyang, Menglun, Mengla, Shangyong and Manggao. The area is renowned for its high orchid diversity: Liu et al. (2015) identified 426 orchid species from 115 genera in 112 this area.

114 N) (Fig. S1), which contains around 60 orchid species (Liu et al. unpublished data). Because about one third of these species are extremely rare (less than two individuals) (Liu et al. 2015), 
mycorrhizal associations were investigated in 44 species belonging to 25 genera for which more

117 than four individuals could be found in the study area. These orchid species include 13 terrestrial,

11817 epiphytic, 6 lithophytic, and 8 species displaying both an epiphytic and lithophytic life form

119 (Table S1). For each orchid species, four to five individual plants were selected. Individuals of a

120 single species were selected in such a way that they were at least $15 \mathrm{~m}$ apart. Orchid species that were both epiphytic and lithophytic were collected separately. In total, 245 plant individuals were sampled (62 terrestrial, 118 epiphytes and 65 lithophytes). For each individual plant, we collected more than 4 independent root fragments (about $2 \mathrm{~cm}$ long) whenever possible without dislodging the plant. Root samples were refrigerated until processing (within 3 days of sampling). Sampled roots were surface-sterilized with ethanol $(70 \%)$ for $30 \mathrm{~s}$ and rinsed three times in sterile water to avoid unnecessary contaminations from the velamen of the roots and surface of root epidermis.

Then the root fragments were checked for the presence of orchid mycorrhizae, that is, intracellular hyphal pelotons (Rasmussen 1995). A 5-mm-long root section harboring pelotons was sampled for each root fragment, that is, five root sections per plant, and stored in $-20{ }^{\circ} \mathrm{C}$ for DNA extraction.

\section{Assessment of mycorrhizal communities}

132 Genomic DNA was extracted from two root sections per orchid individual using the DNeasy 133 PlantMini Kit (Qiagen) following the manufacturer's instructions. To describe the 134 basidiomycetous mycorrhizal community, the effectiveness of several broad-spectrum 135 basidiomycete primer pairs, including ITS1-OF/ITS4-OF (Taylor and McCormick 2008), ITS1136 OF (White et al. 1990) / ITS4-Tul (Taylor and McCormick 2008) and ITS1-OF/ITS4 (White et 

al. 1990) were tested. ITS1-OF and ITS4-OF gave the most consistent amplification with high

138 yields. Clone libraries were constructed following PCR amplification with the primers ITS1-OF 139 and ITS4-OF. PCR conditions were as follows: $94{ }^{\circ} \mathrm{C}$ for $3 \mathrm{~min}$, followed by 32 cycles of $94{ }^{\circ} \mathrm{C}$ 140 for $30 \mathrm{~s}, 52{ }^{\circ} \mathrm{C}$ for $30 \mathrm{~s}$, and $72{ }^{\circ} \mathrm{C}$ for $55 \mathrm{~s}$. The final cycle was followed by an extension of 7$141 \min$ at $72{ }^{\circ} \mathrm{C}$. Clone libraries were constructed for each sample using the following procedure: 142 PCR products were purified using the QIAquick PCR Purification Kit (Qiagen) and cloned using 143 the pGEM-T Easy Vector (TaKaRa, Japan) and competent high DH5 $\alpha$. Ninety-six clones were 144 randomly selected from each library and sequenced using the M13 forward primer. Our previous 145 studies have shown that this was a large enough clonal pool for assessing total species diversity 146 and sequencing completeness (Xing et al. 2015, 2017). All clones were sequenced by Genewiz 147 Inc. (Beijing, China). MEGA6 software (Tamura et al. 2013) was used to align DNA sequences 148 from all the samples. UPARSE (Edgar 2013) was used to group the sequences into operational 149 taxonomic units (OTUs), in which sequences exceeding 97\% homology were clustered into the same OTU. This threshold is the usual proxy for species delimitation among basidiomycetes

151 (Martos et al. 2012, Jacquemyn et al. 2015, 2017). Rarefaction analyses were used to assess completeness of the sequencing. Rarefaction analyses were conducted using EstimateS version 9.0 (Colwell 2013). The different OTUs were identified using the BLAST algorithm and 154 deposited in GenBank (MH005840-MH005926).

\section{Plant ITS amplification and sequencing}

156 From each orchid species, one healthy leaf was selected for genomic DNA extraction. Plant DNA 

2005). The PCR conditions were as follows: $94^{\circ} \mathrm{C}$ for $3 \mathrm{~min}$, followed by $94^{\circ} \mathrm{C}$ for $30 \mathrm{~s}, 55^{\circ} \mathrm{C}$ for $30 \mathrm{~s}$ and $72^{\circ} \mathrm{C}$ for $55 \mathrm{~s}, 32$ cycles, and extension at $72^{\circ} \mathrm{C}$ for $7 \mathrm{~min}$. Amplification products were checked by electrophoresing on a $1.0 \%$ agarose gel to ensure that a single DNA band of the expected size was produced. For sequencing, a QIAquick PCR purification kit (Qiagen, Germany) was used to purify PCR products from unincorporated nucleotides, excess primer and salts, as well as primer dimers. Purified PCR products were sequenced by GENEWIZ Inc. (Beijing, China).

\section{Data analysis}

To compare the phylogenetic diversity of fungal associations between orchid species, we first constructed a ML tree for all the fungal OTUs identified in this research. The 87 fungal OTU sequences were aligned using Clustal X version 2.0 (Larkin et al. 2007). The T92+G model of evolution was identified as the best-fit model for the fungal OTU dataset using the Akaike Information Criterion implemented in jModelTest 2 (Darriba et al. 2012). The ML phylogeny was constructed with RAxML 7.2.8 (Stamatakis et al. 2008). Clade support was estimated with RAxML through a nonparametric bootstrap analysis of 1,000 pseudo-replicate datasets. phylogenetic diversity (PD; Faith 1992) and mean pairwise distance (MPD; Webb et al. 2002) of the OTUs associated with each orchid species. All calculations were done using the software package 'picante' (Kembel et al. 2010) in R (R Development Team 2016). Univariate analysis of 
variance (ANOVA) was used to test the hypothesis that the number of OTUs, phylogenetic diversity (PD) and the mean pairwise distance (MPD) differed significantly between terrestrial,

181 epiphytic and lithophytic orchids.

\section{Network architecture}

To describe the properties of the interaction network, we first assembled all interactions between orchid species and fungal OTUs based on individual occurrences of fungal OTUs on orchid roots and applied all subsequent network analysis to the species-level matrix. Two frequently used network measures were used to describe the architecture of the network: nestedness and modularity. We used a nestedness metric that is based on overlap and decreasing fill (NODF) to calculate the degree of nestedness. This measure is less dependent on the size of the shape of the interaction matrix than other measures of nestedness and therefore provides an unbiased measure to estimate the degree of nestedness (Almeida-Neto et al. 2008). To assess the significance of nestedness, two different null models were used (Guimarães and Guimarães 2006). In the first null model, each cell in the interaction matrix has the same probability of being occupied. This null model is very general and does not take into account the fact that the number of connections

194 per species may vary substantially. A more conservative null model would therefore be a model in which the probability of drawing an interaction is proportional to the degree of specialization (Bascompte et al. 2003). In this null model, the probability of each cell being occupied is the average of the probabilities of occupancy of its row and column (Almeida-Neto et al. 2008). All nestedness analyses were performed using the software package ANINHADO 3.0 (Guimarães and Guimarães 2006). 

annealing algorithm developed by Guimerà and Amaral (2005), which identifies modules whose nodes have the majority of their links inside their own module. This algorithm provides an index of modularity $M$ :

$$
M=\sum_{s=1}^{N_{M}}\left[\frac{l_{s}}{L}-\left(\frac{d_{s}}{2 L}\right)^{2}\right]
$$

where $N_{M}$ is the number of modules, $L$ represents the number of links in the network, $l_{s}$ is the number of links between nodes in module $s$, and $d_{s}$ is the sum of the number of links of the nodes in module $s$. This measure of modularity has been used before to describe the properties of bipartite networks (e.g. Olesen et al. 2007, Fortuna et al. 2010, Thébault and Fontaine 2010). To determine the significance of the observed modularity index, 999 random networks with the same species degree distribution as the original network were constructed and the observed modularity index was compared with indices from random networks (Guimerà et al. 2004).

\section{Phylogenetic constraint analysis}

Finally, we used a phylogenetic signal strength to test whether the phylogenetic relatedness of orchid species correlated with a similar set of mycorrhizal fungi, that is whether the observed interaction network structure was significantly affected by the phylogeny of the plants or the fungi. The fungi that were involved in the studied orchids mainly belonged to three fungal clades

217 (Tulasnellaceae, Ceratobasidiaceae and Sebacinales), and have been called rhizoctonia for 218 convenience (Dearnaley et al. 2012). For better understanding the interaction network of orchid 219 and mycorrhizal fungi, we only used the rhizoctonia dataset (53 OTUs) for further analysis. 
lengths) estimated by phylogenetic inferences, we constructed a ML tree for the orchid species

222 and the rhizoctonia fungi, respectively. Branch lengths were estimated without a molecular clock

223 assumption in the ML trees. The ITS sequences of 40 orchid species and 39 Tulasnellaceae OTUs

224 were aligned using Clustal $\mathrm{X}$ version 2.0 (Larkin et al. 2007). The $\mathrm{K} 2+\mathrm{G}+\mathrm{I}$ and $\mathrm{K} 2+\mathrm{G}$ evolution

225 models were identified as the best-fit models for the orchids and Tulasnellaceae datasets,

226 respectively, using the Akaike Information Criterion implemented in jModelTest 2 (Darriba et al.

227 2012). For both data sets, an ML phylogeny was constructed with RAxML 7.2.8 (Stamatakis et

228 al. 2008). Clade support was estimated with RAxML through a nonparametric bootstrap analysis

229 of 1,000 pseudo-replicate data sets. We then evaluated the strength of the phylogenetic signals of

230 the two phylogenies on the orchids-Tulasnellaceae fungi interaction network using a linear model

231 approach that fits the phylogenetic variance-covariance matrix to the plant-fungi interaction

232 matrix (Ives and Godfray 2006). We applied the phylogenetic bipartite linear model of Ives and

233 Godfray (2006). We calculated the independent phylogenetic signals of the orchids $\left(d_{0}\right)$ and

234 Tulasnellaceae $\left(d_{\mathrm{T}}\right)$ phylogenies on the interaction matrix and the strength of the signal of both

235 phylogenies combined $\left(\mathrm{MSE}_{d}\right)$. The significance of the phylogenetic structure was determined by

236 comparing the mean square error (MSE) of this model of evolution (MSE ${ }_{d}$ ) with the MSE derived

237 under the assumption of no phylogenetic signals (i.e., a star phylogeny) and with the MSE derived

238 under the assumption of a maximum phylogenetic signal (i.e., Brownian motion evolution,

$239 \mathrm{MSE}_{b}$ ). The model minimizing the MSE was considered the best fit. Bipartite linear models were

240 performed using the pblm function in the picante R package (Kembel et al. 2010). 


\section{$241 \quad$ Results}

\section{Fungal diversity}

243 In all orchid species investigated, typical characteristics of orchid mycorrhiza were observed in 244 the roots and for each species one or more ITS sequences were obtained, resulting in a total of 2451343 diverging sequences. Almost all of the obtained sequences corresponded to basidiomycete 246 fungi (1324 sequences), except for a few sequences that belonged to ascomycete fungi (19 247 sequences). The 1324 basidiomycete sequences yielded a total of 87 OTUs at a sequence 248 similarity threshold of $97 \%$ (Table S2). Rarefaction analysis showed that the curve quickly 249 reached an asymptote for the analyzed sequences (Fig. S2). Among them, 53 OTUs (1157 250 sequences) were assigned to rhizoctonia fungi according to Dearnaley et al. (2012). Thirty-nine 251 OTUs (1065 sequences), 11 OTUs (64 sequences) and 3 OTUs (28 sequences) were assigned to members of Tulasnellaceae, Ceratobasidiaceae and Sebacinales, respectively. Besides, other

253 fungal taxa known to associate with orchids were retrieved, including members of the 254 Thelephoraceae (2 OTUs, 5 sequences), Cortinariaceae (2 OTUs, 24 sequences), Marasmiaceae 255 (1 OTU, 10 sequences), Russulaceae (1 OTU, 3 sequences), unknown Cantharellales (4 OTUs, 25616 sequences), and Atractiellales (3 OTUs, 21 sequences). Additionally, a number of possibly 257 endophytic fungi belonging to Tricholomataceae and Septobasidiaceae were only sporadically 258 detected.

259 Epiphytic orchids associated with 50 different OTUs, whereas terrestrial and lithophytic 260 orchids associated with 25 and 24 OTUs, respectively (Table S1; Fig. S3). When comparing 261 fungal communities between terrestrial, epiphytic and lithophytic orchids, it is apparent that 
members of the Tulasnellaceae were the dominant species in all life forms. However, the relative frequency of Tulasnellaceae increased from terrestrial (47.33\%), over epiphytic $(83.87 \%)$ to lithophytic orchids (96.71\%) (Fig. 1). Moreover, distinct guilds of fungal OTUs associated with the different life forms of orchids. Out of 87 OTUs, no OTU was shared between the three life forms, indicating that they associate with distinct mycorrhizal fungi. Epiphytic and lithophytic orchids shared one OTU with terrestrial orchids, whereas epiphytic and lithophytic orchids shared 10 OTUs (Fig. S3). Orchid species that occurred both in epiphytic and lithophytic habitats associated with 29 different OTUs in total, of which eight OTUs were shared between the two life forms. On average, $42.1 \%$ of all OTUs found in an orchid species displaying both life forms were shared between the epiphytic and the lithophytic life form.

The average number of fungal OTUs retrieved per orchid species did not differ significantly $(P>0.05)$ between life forms (Fig. 2a). Epiphytic orchids interacted on average with $3.36 \pm 0.50$ OTUs, whereas terrestrial and lithophytic orchids associated with $2.69 \pm 0.36$ and $2.85 \pm 0.40$ OTUs. Average phylogenetic diversity, on the other hand, was highest in terrestrial orchids (PD $=0.6252 \pm 0.0545)$ and was significantly $(P<0.05)$ higher than that of lithophytes $(0.4921 \pm$ 0.0413), but not of that of epiphytes $(0.6018 \pm 0.0592)$ (Fig. 2b). Finally, the highest MPD was detected in the epiphytic orchids $(0.3491 \pm 0.0636)$, but it was not significantly higher than that observed in terrestrial $(0.3212 \pm 0.0823)$ or lithophytic orchids $(0.2154 \pm 0.0548)$ (Fig. 2c).

\section{Nestedness and modularity}

The overall network comprised 52 orchid species (13 terrestrial, 17 epiphytic, 6 lithophytic, and 8 species displaying both an epiphytic and lithophytic life form) and 87 fungal OTUs, and showed 
$284<0.01)$ nested $\left(\mathrm{NODF}=5.53, E_{r}=3.98, C_{e}=4.82\right)$. The modularity analysis indicated that the 285 network was significantly modular $\left(M=0.7389, M_{\text {random }}=0.6998\right)$ and that 15 distinct modules 286 were identified (Fig. 3). These modules had, on average, 9 links within modules and 1.6 links to 287 other modules. The largest module consisted of 10 orchid species and contained eight epiphytic 288 and two lithophytic orchids. The second largest module contained eight orchid species, five of 289 which were lithophytic orchids and three epiphytic species. Terrestrial orchids formed a set of 290 five distinct modules, which had almost no links to modules containing epiphytic or lithophytic orchids (Fig. 3).

When only rhizoctonia fungi were considered, very similar results were obtained, indicating that results are not biased due to sporadic occurrence of non-rhizoctonia fungi. In this case, the interaction network consisted of 52 orchid species and 53 OTUs and contained 117 binary links $(C=0.043)$ (Fig. 4). The network was again significantly $(P<0.05)$ nested $\left(\mathrm{NODF}=7.78, E_{r}=\right.$ 4.79, $\left.C_{e}=6.10\right)$. The modularity index was high $(M=0.7128)$, and significantly larger than that of random matrices $\left(M_{\text {random }}=0.685 \pm 0.013\right)$. There were 15 modules that varied in size between 1 and 9 orchids (average number of orchid species within a module: 3.5) (Figure S4). Modules containing terrestrial orchids were almost completely isolated from modules containing epiphytic or lithophytic orchids and vice versa. Modules containing epiphytic orchids contained lithophytic orchids, confirming our previous analyses that epiphytic and lithophytic orchids share some of their fungal partners and that they are grouped in several modules (Fig. S4).

\section{Phylogenetic signal}


Finally, we used a linear model approach to evaluate the phylogenetic signal of both the orchid and the fungal phylogenies on the orchid-rhizoctonia network. For both the orchids and the fungi, the phylogenetic signal was very weak $\left(d_{\mathrm{o}}<0.001,95 \%\right.$ CI $0-0.023 ; d_{\mathrm{f}}<0.001,95 \%$ CI $0-6.612 \mathrm{e}-$ 06). The strength of the overall phylogenetic signal $\left(\mathrm{MSE}_{d}=0.044\right)$ was similar to that of a star phylogeny $\left(\mathrm{MSE}_{s}=0.044\right)$ and lower than that of the maximal inertia $\left(\mathrm{MSE}_{b}=0.0471\right)$. Therefore, neither phylogenetic relationships among orchids nor among fungi imposed some structure on the association matrix (Fig. 4). When we analyzed the phylogenetic signal of the orchid-rhizoctonia subnetworks in different life forms, the phylogenetic signal was small and not significantly different from zero for lithophytic $\left(d_{0}=0.1411,[0.04-0.22] ; d_{f}<0.001,[0-0.020]\right)$, epiphytic $\left(d_{\mathrm{o}}<0.001,[0-0.002] ; d_{\mathrm{f}}<0.001,[0-0.001]\right)$ and terrestrial orchids $\left(d_{\mathrm{o}}=0.007,[0-\right.$ 0.209]; $d_{\mathrm{f}}<0.001$, [0-0.031]). Similar results were obtained when only Tulasnella fungi were taken into account (data not shown).

\section{Discussion}

Non-random associations have been commonly observed in plant-fungus interaction networks (e.g. Montesinos-Navarro et al. 2012; Chagnon et al. 2012, Martos et al. 2012, Toju et al. 2014; 2016; Bahram et al. 2015), but the precise mechanisms leading to non-random interactions are less well understood. Here, we investigated the architecture of the network of interactions between a large number of orchids and mycorrhizal fungi and asked whether the observed patterns were the result of ecological, evolutionary and/or co-evolutionary processes. Our analyses showed that the observed interaction network was significantly modular and to a much lesser extent nested. Terrestrial and epiphytic/lithophytic orchids clearly associated with distinct sets of mycorrhizal 

partner selectivity and high turnover of mycorrhizal partners were the main factors explaining the observed network architecture.

\section{Host specificity and selectivity in terrestrial, epiphytic and lithophytic orchid species}

330 Within tropical ecosystems, orchids with different life forms (in this case terrestrial, epiphytic and

331 lithophytic orchids) are capable of occupying different niches and therefore coexisting in one habitat. Because the three life forms represent largely different environments, they may select for different mycorrhizal fungi. Our results showed that members of the Tulasnellaceae were the most dominant fungi in the three life forms, supporting previous findings that Tulasnellaceae symbionts are ubiquitous in terrestrial orchids worldwide (Jacquemyn et al. 2017) as well as in some epiphytic orchids (Kartzinel et al. 2013, Xing et al. 2017). Besides members of the Tulasnellaceae, other rhizoctonia fungi of the Ceratobasidiaceae and Sebacinales (Dearnaley et al. 2012) were observed, particularly in terrestrial and epiphytic orchids, but not in the lithophytic species, which almost exclusively associated with fungi from the Tulasnellaceae. Terrestrial orchids further had sporadic associations with members of Thelephoraceae, Cortinariaceae, Marasmiaceae, unknown Cantharellales and Atractiellales.

The factors that drive mycorrhizal specificity are not clear, but it has been suggested that it might be affected by environmental factors (Jacquemyn et al. 2010, Kartzinel et al. 2013).

344 Associating with multiple fungi could confer symbiotic assurance when mycorrhizal fungi show 345 a patchy distribution or are only stochastically available, which may be crucial in dynamic or 346 disturbed habitats such as forest canopies (Kartzinel et al. 2013). Although the number of fungal 
associates did not significantly differ between life forms, terrestrial and epiphytic orchids

348 interacted with more diversified fungi than lithophytic orchids and an increasing specificity

349 towards Tulasnellaceae fungal associates was observed from terrestrial to epiphytic and

350 lithophytic orchids. Epiphytic orchids showed levels of phylogenetic diversity similar to that of

351 terrestrial orchids. Compared to terrestrial and epiphytic habitats, lithophytic habitats represent

352 harsh environments that are characterized by lower availability of above-ground water and

353 nutrient supplies, which may explain the increased dependency and specificity on mycorrhizal

354 fungi. Moreover, lithophytic habitats most likely also not support fungi that are involved in

355 associations with other organisms such as trees. The lower phylogenetic diversity of mycorrrhizal

356 fungi observed in lithophytic orchids therefore most likely arises from ecological factors

357 associated with lithophytic habitats.

\section{Network architecture}

359 Extreme host selectivity and specialization may lead to significant turn-over in orchid-fungus associations in co-occurring orchid species and therefore explain the low nestedness values that were observed in this study and the significant modularity that is typically found in orchid-fungus networks (Jacquemyn et al. 2015). Our results showed that some orchids (e.g. Nervilia plicata, Oberonia variabilis and Epigeneium amplum) associated with only a single fungal taxon, confirming previous research that extreme host specialization can be observed in orchids (e.g. majority of the orchid species investigated here interacted with several partners at the same time, 
indicating that extreme host specialization cannot be the sole explanation for the observed network structure.

One possible other explanation for the observed variation in network structure is based on the concept of forbidden links, i.e. ecological constraints that prevent the occurrence of certain pairwise interactions among those possible in the entire network (Jordano et al. 2006, Olesen et al. 2011). Our results indicated very little overlap in fungal associations between terrestrial orchids on the one hand and epiphytic/lithophytic orchids on the other hand. The observed differences in mycorrhizal partners between life forms suggest a clear ecological barrier between terrestrial and epiphytic/lithophytic habitats, which is somewhat similar to temporal uncoupling therefore explain the strong modular structure when all interactions are analyzed across different life forms. Strong evidence of modularity was also found in a mycorrhizal network of orchids on organize orchid-fungus interactions do not depend on orchid life form and that within each life form link specificity (Lewinsohn et al. 2006) and strong turnover in mycorrhizal partners have further contributed to the observed modular structure of the entire network. Similar patterns have 
been found for orchid species in species-rich Mediterranean orchid communities (Jacquemyn et

390 al. 2015) and may reflect spatial or mutual selective limitations acting between different orchids

391 and fungi (Jacquemyn et al. 2012, 2014). Strong turnover in mycorrhizal partners may decrease

392 resource competition and therefore lead to niche partitioning and stable coexistence of multiple

393 orchid species (e.g. Jacquemyn et al. 2014; 2015). Seed germination experiments have indeed

394 shown that strong partner selectivity and non-random spatial distribution of mycorhizal fungi in

395 the soil leads to stable co-existence of orchid species (Jacquemyn et al. 2012, 2014; Waud et al.

396 2016).

\section{Phylogenetic constraints}

398 Apart from differences in ecological conditions, the architecture of interaction networks can also

399 be determined by phylogenetic relationships between species (Bascompte and Jordano 2007). In

400 this case, it can be expected that phylogenetically closely related species tend to exhibit similar

401 physiological or ecological properties and therefore may have similar network properties

402 (Freckleton et al. 2002, Blomberg et al. 2003, Garland et al. 2005, Ives and Godfray 2006).

403 Previous research has shown that in several orchid genera closely related species associated with

404 more similar fungal communities (e.g. Cypripedium (Shefferson et al. 2007), Goodyera

405 (Shefferson et al. 2010), Orchis (Jacquemyn et al. 2011), Dendrobium (Xing et al. 2017),

406 suggesting that phylogenetic constraints may influence the mycorrhizal community an orchid

407 associates with. However, in this research no phylogenetic signal on the overall network structure

408 was detected. These results are in line with findings of Martos et al. (2012), who also showed a 
weak overall phylogenetic signal in the interaction matrix of a large number of tropical orchids and their associated mycorrhizal fungi.

Our results contrast with those from Martos et al. (2012), in that no strong phylogenetic signal of both partners was found in the subnetworks of any of the life forms studied. The stronger phylogenetic signal in the epiphytic sub network of Martos et al. (2012) might be explained by the phylogenetic depth of epiphytic orchid taxa on Réunion Island. Most orchids belonged to the sub tribe Angraecinae, which diversified in Madagascar and the Indian Ocean islands, whereas at our study site, the sampled orchids were a more phylogenetically diverse assemblage of epiphytic orchids on the one hand, and terrestrial orchids on the other hand.

\section{Conclusions}

To conclude, our results showed that orchids displaying different life forms associated with different fungal symbionts, which resulted in a network structure that was significantly modular. Within life forms, multiple modules were found, suggesting that the processes that organize orchid-fungus interactions are independent of life form. Our results further showed an increasing specificity towards Tulasnellaceae fungi from terrestrial over epiphytic to lithophytic orchids, suggesting that more stressful environments limit the potential pool of mycorrhizal partners and thus the potential for associations with diverse fungi. Significant modularity in the network may point to a high interaction intimacy between orchids and fungi and a strong ecological barrier between terrestrial and epiphytic/lithophytic habitats. Phylogenetic relationships, on the other hand, did not affect network patterns, indicating that ecological factors were more important than past evolutionary history in explaining the observed network architecture. To gain better insights 

into the precise mechanisms leading to the modular structure, we eagerly anticipate future studies

431 that describe the total pool of mycorrhizal symbionts occurring in different habitats and compare

432 patterns of partner choice between orchids with different life forms using seed germination 433 experiments and fungal identifications.

434 
436

Almeida-Neto, M. et al. 2008. A consistent metric for nestedness analysis in ecological systems: reconciling concept and measurement. - Oikos 117: 1227-1239.

Bahram, M. et al. (2014) Network perspectives of ectomycorrhizal associations. - Fungal Ecol. 7: 70-77.

Bascompte, J. 2010. Structure and dynamics of ecological networks. - Science 329: 765-766.

Bascompte, J. and Jordano, P. 2014. Mutualistic networks. Monographs in population biology. - Princeton Univ. Press.

Bascompte, J. and Jordano, P. 2007. Plant-animal mutualistic networks: the architecture of biodiversity. Ann. Rev. Ecol. Syst. 38: 567-593.

Bascompte, J. et al. 2003. The nested assembly of plant-animal mutualistic networks. - Proc. Natl. Acad. Sci. USA 100: 9383-9387.

Blomberg, S.P. et al. 2003. Testing for phylogenetic signal in comparative data: behavioral traits are more labile. - Evolution 57: 2147-2156.

Bonfante, P. and Genre, A. 2010. Mechanisms underlying beneficial plant-fungus interactions in mycorrhizal symbiosis. - Nat. Commun. 1: 48.

Cameron, K.M. 2005. Leave it to the leaves: a molecular phylogenetic study of Malaxideae (Epidendroideae, Orchidaceae). - Am. J. Bot. 92: 1025-1032.

Chagnon, P.L. et al. (2012) Using ecological network theory to arbuscular mycorhizal fungi - plant interactions: the importance of basic assumptions. - New Phytol. 194: 307-314.

Chagnon, P.L. (2016) Seeing networks for what they are in mycorrhizal ecology. - Fungal Ecol. 24: 148154.

Colwell, R.K. 2013. EstimateS: Statistical estimation of species richness and shared species from samples. Version 9. User's Guide and application published at: http://purl.oclc.org/estimates

Darriba, D. et al. 2012. jModelTest 2: more models, new heuristics and parallel computing. - Nat. Methods 9: 772

Dearnaley, J.W.D. et al. 2012. Orchid mycorrhizas: molecular ecology, physiology, evolution and conservation aspects. In: Hock B, ed. Fungal associations, 2nd edn. Berlin, Germany: Springer-Verlag, 207-230.

Donatti, C.I. et al. 2011. Analysis of a hyper-diverse seed dispersal network: modularity and underlying mechanisms. - Ecol. Lett. 14:773-781.

Edgar, R.C. 2013. UPARSE: highly accurate OTU sequences from microbial amplicon reads. - Nat. Methods 10: 996-998.

Faith, D.P. 1992. Conservation evaluation and phylogenetic diversity. - Biol. Conserv. 61: 1-10.

Fortuna, M.A. et al. 2010. Nestedness versus modularity in ecological networks: two sides of the same coin. - J. Anim. Ecol. 79: 811-817.

Freckleton, R.P. et al. 2002. Phylogenetic analysis and comparative data: a test and review of evidence. Am. Nat. 160: 712-726.

Garland, T. et al. 2005. Phylogenetic approaches in comparative physiology. - J. Exp. Biol. 208: 30153035.

Gravendeel, B. et al. 2004. Epiphytism and pollinator specialisation: drivers for orchid diversity? - Philos. Trans. R. Soc. of Lond. B. Biol Sci. 359: 1523-1535.

Guimarães, P.R. adn Guimarães, P. 2006. Improving the analyses of nestedness for large sets of matrices. - Environ. Modell. Softw. 21: 1512-1513. 
Guimerà, R. and Amaral, L.A.N. 2005. Functional cartography of complex metabolic networks. - Nature 433: 895-900.

Guimerà, R. et al. 2004. Modularity from fluctuations in random graphs and complex networks. - Phys. Rev. E. 70: 025101.

Ives, A.R. and Godfray, H.C. 2006. Phylogenetic analysis of trophic associations. - Am. Nat. 168: E1-E14. Jacquemyn, H. et al. 2012. Spatial variation in belowground seed germination and divergent mycorrhizal associations correlate with spatial segregation of three co-occurring orchid species. - J. Ecol. 10: $1328-1337$.

Jacquemyn, H. et al. 2014. Co-existing orchid species have distinct mycorrhizal communities and display strong spatial segregation. - New Phytol. 202: 616-627.

Jacquemyn, H. et al. 2015. Mycorrhizal networks and coexistence in species-rich orchid communities. New Phytol. 206: 1127-1134.

Jacquemyn, H. et al. 2017. Biogeography of Orchid Mycorrhizas. In: Tedersoo L. (eds) Biogeography of Mycorrhizal Symbiosis. Ecological Studies (Analysis and Synthesis), vol 230. Springer, Cham

Jacquemyn, $\mathrm{H}$ et al. 2010. Low specificity and nested subset structure characterize mycorrhizal associations in five closely related species of the genus Orchis. - Mol. Ecol. 19: 4086-4095.

Jacquemyn, H. et al. 2011. Analysis of network architecture reveals phylogenetic constraints on mycorrhizal specificity in the genus Orchis (Orchidaceae). - New Phytol. 192: 518-528.

Jordano, P. et al. 2006. The ecological consequences of complex topology and nested structure in pollination webs. In: Waser, N.M. and Ollerton, J. (eds.) Plant-Pollinator Interactions. From Specialization to Generalization. University of Chicago Press, Chicago. pp. 173-199.

Jordano, P. et al. 2003. Invariant properties in coevolutionary networks of plant-animal interactions. - Ecol. Lett. 6: 69-81.

Kartzinel, T.R. et al. 2013. Highly diverse and spatially heterogeneous mycorrhizal symbiosis in a rare epiphyte is unrelated to broad biogeographic or environmental features. - Mol. Ecol. 22: 5949-5961.

Kembel, S.W. et al. 2010. Picante: R tools for integrating phylogenies and ecology. - Bioinformatics 26 : 1463-1464.

Larkin, M.A. et al. 2007. Clustal W and Clustal X version 2.0. - Bioinformatics 23: 2947-2948.

Lewinsohn, T.M. et al. 2006. Structure in plant-animal interaction assemblages. - Oikos 113: 174-184.

Liu, Q. et al. 2015. Orchid conservation in the biodiversity hotspot of southwestern China. - Conserv. Biol. 29: $1563-1572$.

Martos, F. et al. 2012. The role of epiphytism in architecture and evolutionary constraint within mycorrhizal networks of tropical orchids. - Mol. Ecol. 21: 5098-5109.

McCormick, M.K. and Jacquemyn, H. 2014. What constrains the distribution of orchid populations? - New Phytol. 202: 392-400.

McCormick, M.K. et al. 2012. Limitations on orchid recruitment: not a simple picture. -Mol. Ecol. 21: 1511-1523.

Montesinos-Navarro, A. et al. (2012) The network structure of plant-arbuscular mycorrhizal fungi. - New Phytol. 194: 536-547.

Olesen, J.M et al. 2007. The modularity of pollination networks. - Proc. Natl. Acad. Sci. USA 104: 1989119896.

Olesen,J.M. et al. 2011. Missing and forbidden links in mutualistic networks. - Proc. R. Soc. B 278: 725732. 
Põlme, S. et al. 2018. Host preference and network properties in biotrophic plant-fungal associations. New Phytol. 217: 1230-1239.

Rasmussen, H.N. (1995). Terrestrial orchids: from seed to mycotrophic plant. Cambridge University Press, New York

Rasmussen, H.N., and Rasmussen, F.N. 2009. Orchid mycorrhiza: implications of a mycophagous life style. - Oikos 118: 334-345.

Rezende, E.L. et al. 2009. Compartments in a marine food web associated with phylogeny, body mass, and habitat structure. - Ecol. Lett. 12: 779-788.

Rezende, E.L. et al. 2007. Effects of phenotypic complementarity and phylogeny on the nested structure of mutualistic networks. - Oikos 11: 1919-1929.

Shefferson, R.P. et al. 2010. Evolution of host breadth in broad interactions: mycorrhizal specificity in East Asian and North American rattlesnake plantains (Goodyera spp.) and their fungal hosts. - Mol. Ecol. 19: 3008-3017.

Shefferson, R.P. et al. 2007. The evolutionary history of mycorrhizal specificity among lady's slipper orchids. - Evolution 61: 1380-1390.

Shefferson, R.P. et al. 2005. High specificity generally characterizes mycorrhizal association in rare lady's slipper orchids, genus Cypripedium. - Mol. Ecol. 14: 613-626.

Smith, S.E. and Read, D.J. (2008). Mycorrhizal symbiosis. Cambridge, UK: Academic Press.

Stamatakis, A. et al. 2008. A rapid bootstrap algorithm for the RAxML web servers. - Syst. Biol. 57: 758771.

Swarts, N.D. et al. 2010. Ecological specialization in the orchid mycorrhizal interaction leads to rarity in the endangered terrestrial orchid Caladenia huegelii. - Mol. Ecol. 19: 3226-3242.

Tamura, K. et al. 2013. MEGA6: Molecular evolutionary genetics analysis version 6.0. - Mol. Biol. Evol. 30: $2725-2729$.

Taylor, D.L. and Bruns, T.D. 1997. Independent, specialized invasions of ectomycorrhizal mutualism by two nonphotosynthetic orchids. - Proc. Natl. Acad. Sci. USA 94: 4510-4515.

Taylor, D.L. and McCormick, M.K. 2008. Internal transcribed spacer primers and sequences for improved characterization of basidiomycetous orchid mycorrhizas. - New Phytol. 177: 1020-1033.

Thébault, E. and Fontaine, C. (2010). Stability of ecological communities and the architecture of mutualistic and trophic networks. - Science 329: 853-856.

Toju, H. et al. 2014. Assembly of complex plant-fungus networks. - Nat. Commun. 5: 5273.

Toju, H. et al. 2016. Ericaceous plant-fungus networks in a harsh alpine-subalpine environment. - Mol. Ecol. 25: 3242-3257.

van der Heijden, M.G.A. et al. 2015. Mycorrhizal ecology and evolution: the past, the present, and the future. - New Phytol. 205: 1406-1423.

Warcup, J.H. 1985. Rhizanthella gardneri (Orchidaceae), its rhizoctonia endophyte and close association with Melaleuca uncinata (myrtaceae) in western Australia. - New Phytol. 99: 273-280.

Waterman, R.J. et al. 2011. The effects of above- and belowground mutualisms on orchid speciation and coexistence. - Am. Nat. 177: E54-E68.

Waud, M. et al. 2016. Specificity and localised distribution of mycorrhizal fungi in the soil may contribute to co-existence of orchid species. - Fungal Ecol. 20: 155-165.

Webb, C. et al. 2002. Phylogenies and community ecology. - Annu. Rev. Ecol. Evol. Syst.33: 475-505. 
White, T.J. et al. 1990. Amplification and direct sequencing of fungal ribosomal RNA genes for phylogenetics. In: InnisMA, Gelfand DH, Sninsky JJ, White TJ (eds) PCR protocols: a guide to methods and applications. Academic, San Diego

Xing, X. et al. 2015. Mycorrhizal fungal diversity and community composition in a lithophytic and epiphytic orchid. - Mycorrhiza 25: 289-296.

Xing, X. et al. 2017. Phylogenetic constrains on mycorrhizal specificity in eight Dendrobium (Orchidaceae) species. - Sci. China Life Sci. 60: 536-544.

Zhang, J., and Cao, M. (1995). Tropical forest vegetation of Xishuangbanna, SW China and its secondary changes, with special reference to some problems in local nature conservation. - Biol. Conserv. 73 : $229-238$. 
(a)

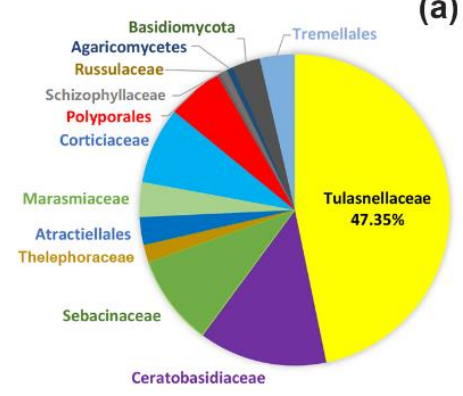

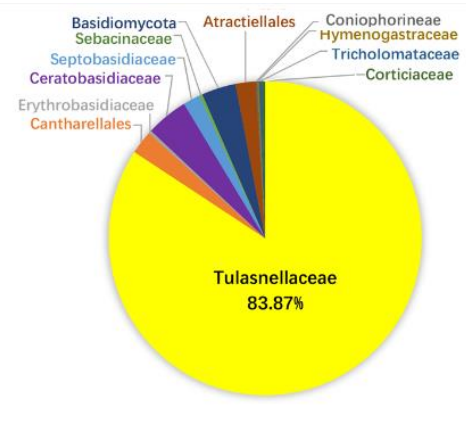

(b)

(c)

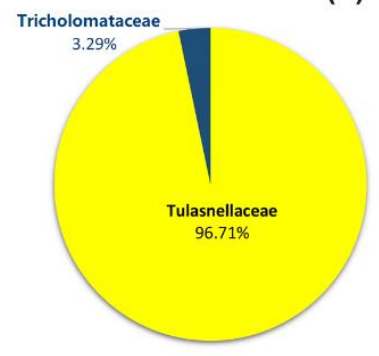

574

575

576

577

578

579

580

581

582

583

584

585

586

587

588

Figure 1. Frequency distribution (based on number of sequences) of fungal families detected in orchids displaying different life forms. (a) Terrestrial orchids; (b) epiphytic orchids; (c) lithophytic orchids.

(c)



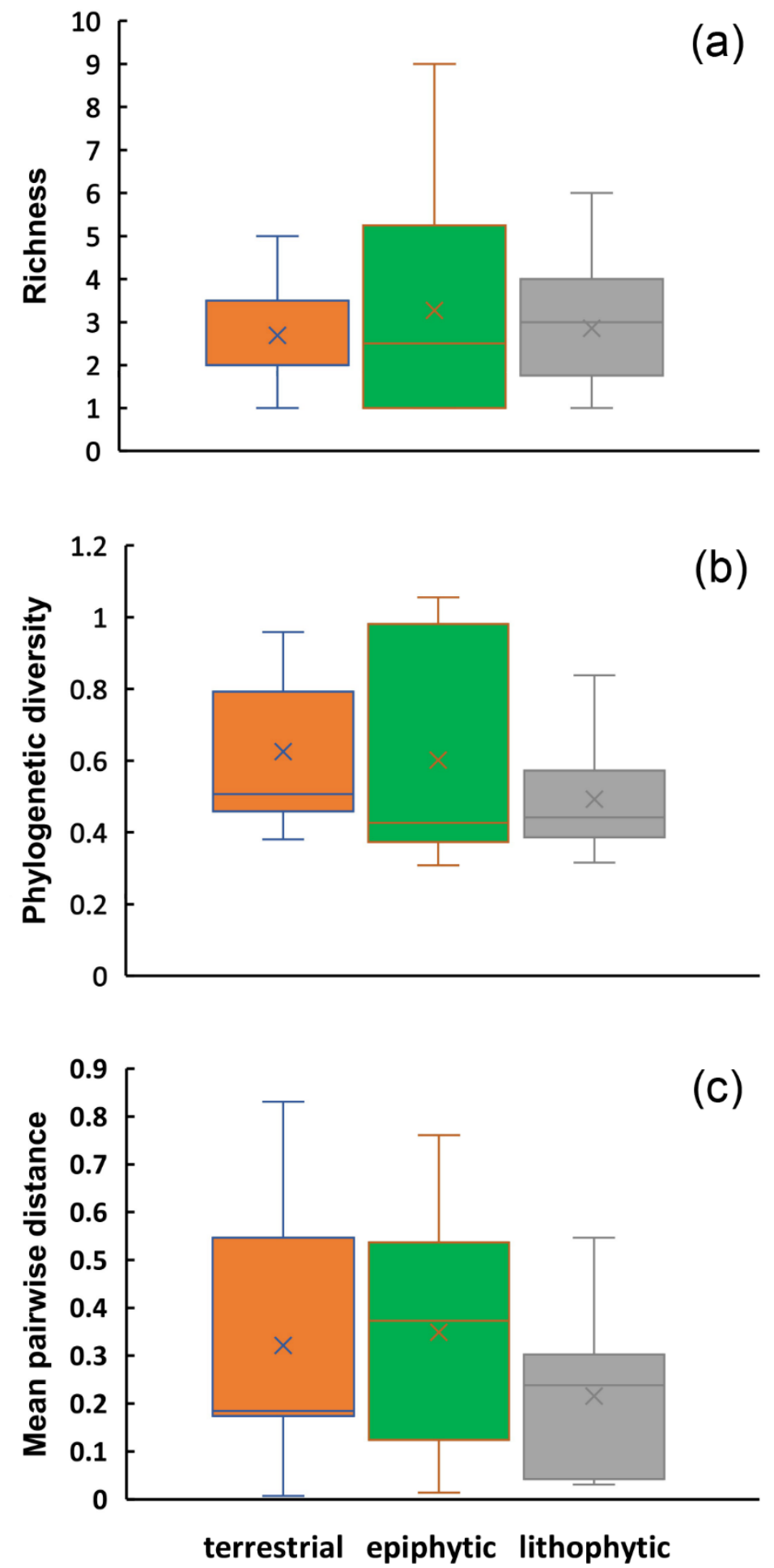

590 Figure 2. Fungal diversity in terrestrial, epiphytic and lithophytic orchids. (a) OTU richness; (b) 591 Phylogenetic diversity; (c) Mean pairwise distance. 


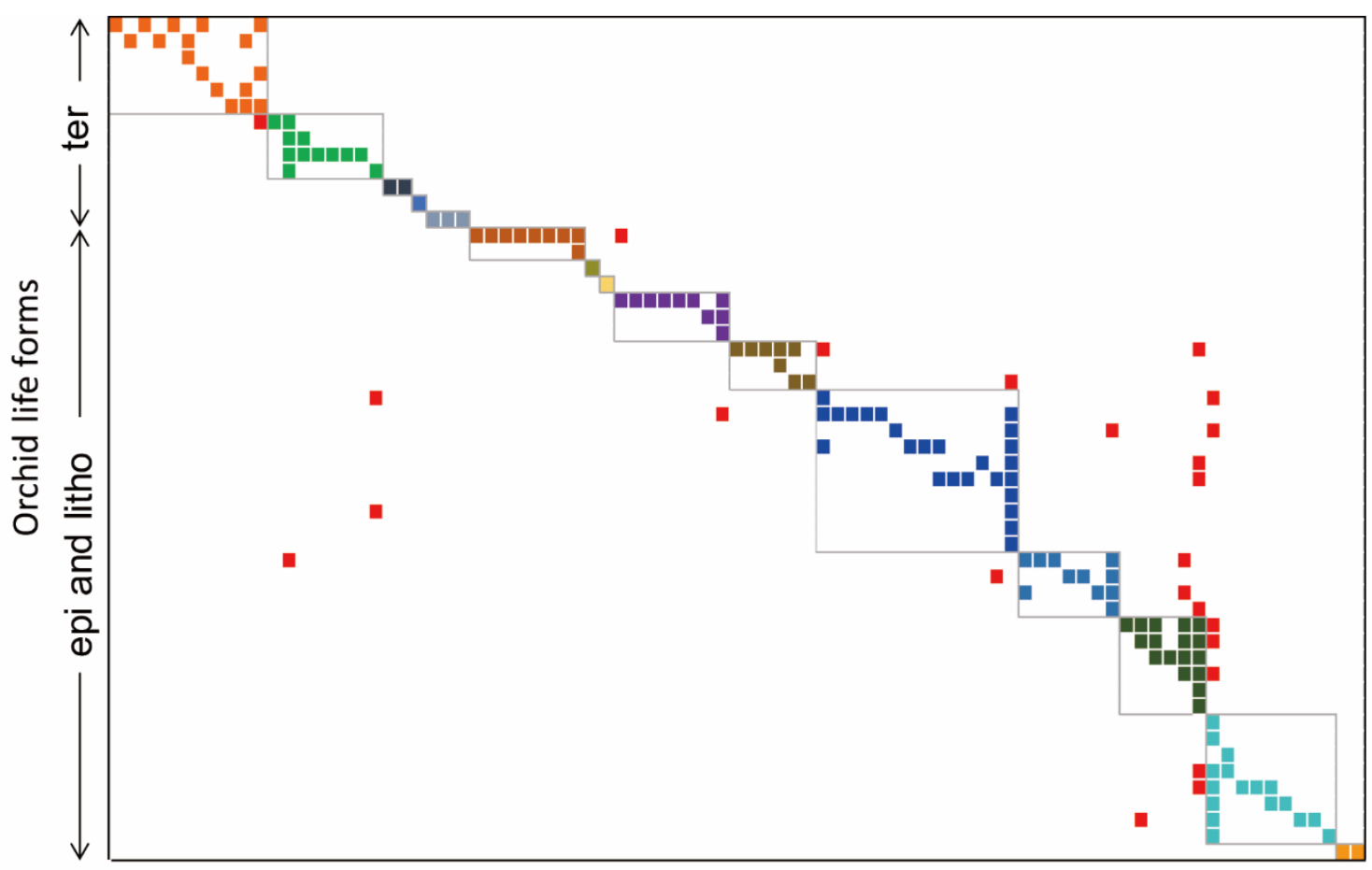

Figure 3. Matrix representation of the interactions between 44 orchid species (including 8 epi/lithophytic species) (columns) and 87 orchid mycorrhizal fungal OTUs (rows). The overall network was significantly modular. The clusters displaying the largest modularity include terrestrial, epiphytic and lithophytic orchid-fungus interactions. The 15 identified modules are shown in different colors. Red cells are species links to other modules, and non-red cells are links within modules. 

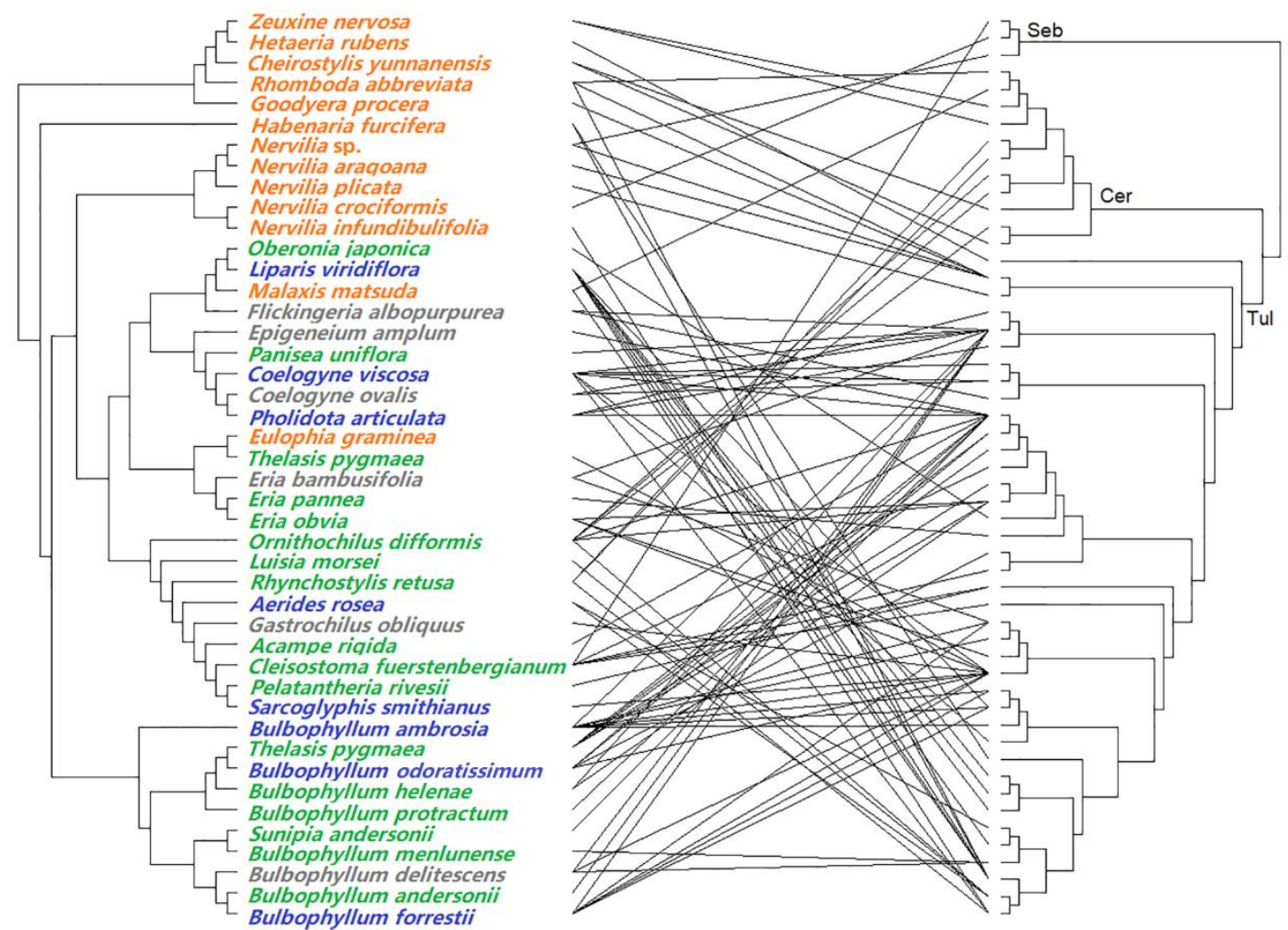

615 Figure 4. Interaction network between orchids and rhizoctonia fungi. The network shows all links 616 between 53 rhizoctonia OTUs and 44 orchid species (13 terrestrial, 17 epiphytic, 6 lithophytic 617 and 8 epiphytic/lithophytic orchids) (103 binary links in total). On the orchids phylogenetic tree, 618 terrestrial, epiphytic, lithophytic and epiphytic/lithophytic are shown in orange, green, grey and 619 blue, respectively. Seb, Sebacinales; Cer, Ceratobasidiaceae; Tul, Tulasnellaceae. 
621 TABLE S1 Different life forms of orchid species collected from Xishuangbanna, Yunnan 622 province, China and their fungal associates.

623 TABLE S2 List of fungal operational taxonomic units (OTU) identified using cloning techniques

624 FIGURE S1 Map of Xishuangbanna showing the National Natural Reserve and Menglun 625 subreserve where 44 orchid species (including 8 epi-/lithophytic species) were sampled.

626 FIGURE S2 Rarefaction analysis performed on the internal transcribed spacer sequence data 627 obtained from the clone libraries for all orchid species (1324 sequences), using a $97 \%$ sequence 628 similarity threshold value.

629 FIGURE S3 Sharing of orchid mycorrhizal OTUs between terrestrial, lithophytic and epiphytic 630 orchids.

631 FIGURE S4 Matrix representation of the interactions between 44 orchid species (including 8 epi632 /lithophytic species) (columns) and 53 rhizoctonia OTUs (rows). The overall network was 633 significantly modular. The clusters displaying the largest modularity include terrestrial, epiphytic 634 and lithophytic orchid-fungus interactions. The 15 identified modules are shown in different 635 colors. Red cells are species links to other modules, and non-red cells are links within modules. 